\title{
Sleep-Dependent Declarative Memory Consolidation- Unaffected after Blocking NMDA or AMPA Receptors but Enhanced by NMDA Coagonist D-Cycloserine
}

\author{
Gordon B Feld*, 1,2, Tanja Lange ${ }^{2}$, Steffen Gais ${ }^{3}$ and Jan Born*,1,4 \\ I'Institute of Medical Psychology and Behavioral Neurobiology, University of Tuebingen, Tuebingen, Germany; ${ }^{2}$ Department of \\ Neuroendocrinology, University of Luebeck, Luebeck, Germany; ${ }^{3}$ Institute of Psychology, Ludwig-Maximilian University, Munich, Germany; \\ ${ }^{4}$ Center for Integrative Neuroscience, University of Tuebingen, Tuebingen, Germany
}

\begin{abstract}
Sleep has a pivotal role in the consolidation of declarative memory. The coordinated neuronal replay of information encoded before sleep has been identified as a key process. It is assumed that the repeated reactivation of firing patterns in glutamatergic neuron assemblies translates into plastic synaptic changes underlying the formation of longer-term neuronal representations. Here, we tested the effects of blocking and enhancing glutamatergic neurotransmission during sleep on declarative memory consolidation in humans. We conducted three placebo-controlled, crossover, double-blind studies in which participants learned a word-pair association task. Afterwards, they slept in a sleep laboratory and received glutamatergic modulators. Our first two studies aimed at impairing consolidation by administering the NMDA receptor blocker ketamine and the AMPA receptor blocker caroverine during retention sleep, which, paradoxically, remained unsuccessful, inasmuch as declarative memory performance was unaffected by the treatment. However, in the third study, administration of the NMDA receptor coagonist D-cycloserine (DCS) during retention sleep facilitated consolidation of declarative memory (word pairs) but not consolidation of a procedural control task (finger sequence tapping). Administration of DCS during a wake interval remained without effect on retention of word pairs but improved encoding of numbers. From the overall pattern, we conclude that the consolidation of hippocampus-dependent declarative memory during sleep relies on NMDA-related plastic processes that differ from those processes leading to wake encoding. We speculate that glutamatergic activation during sleep is not only involved in consolidation but also in forgetting of hippocampal memory with both processes being differentially sensitive to DCS and unselective blockade of NMDA and AMPA receptors.

Neuropsychopharmacology (20I3) 38, 2688-2697; doi:I0.1038/npp.20 13.179; published online 2I August 20I3
\end{abstract}

Keywords: glutamate; NMDA; AMPA; memory consolidation; forgetting; sleep

\section{INTRODUCTION}

Although obscure only a decade ago, the mechanisms of memory maintenance have received growing attention over the past years. This research has led to the conclusion that although memory is most effectively encoded during wakefulness, sleep promotes the consolidation of memory. In the declarative domain, memory consolidation during sleep is thought to be an active process that involves reactivating neuronal ensembles that encoded these memories during wakefulness (Diekelmann and Born, 2010; Oudiette and Paller, 2013). However, it remains unclear which neurochemical processes are mainly involved in

*Correspondence: GB Feld or Professor J Born, Institute of Medical Psychology and Behavioral Neurobiology, University of Tuebingen, Otfried-Müller-Straße 25, Tuebingen, Baden-Würtemberg 72076, Germany, Tel: +49 707| 2988924, Fax: +49 707| 292506, E-mail: jan.born@uni-tuebingen.de or gordon.feld@uni-tuebingen.de Received 25 April 2013; revised 2 July 2013; accepted 23 July 2013; accepted article preview online 26 July 2013 translating memory reactivation into plastic changes (Frank and Benington, 2006).

Work in rodents shows that neuron assemblies that displayed correlated activity during wakefulness are more likely to fire together during subsequent sleep (Wilson and McNaughton, 1994). This replay occurs in the same sequence as during wakefulness and is coordinated between the hippocampus and neocortex (Ji and Wilson, 2007; Skaggs and McNaughton, 1996). A causal role of replay during slow wave sleep (SWS) for the consolidation of hippocampus-dependent declarative memory was demonstrated in humans (Rasch et al, 2007; Rudoy et al, 2009). Reactivation in hippocampal and neocortical networks involves glutamatergic neurons and is temporally coupled to hippocampal sharp wave-ripples and neocortical slow oscillations, both of which support $N$-methyl-D-aspartate (NMDA) and $\alpha$-amino-3-hydroxy-5methyl-4-isoxazolepropionic-acid (AMPA) receptor-related synaptic plasticity (Behrens et al, 2005; Chauvette et al, 2012; Csicsvari et al, 1999; Espinosa and Kavalali, 2009; King et al, 1999). 
Long-term potentiation (LTP) is considered a candidate plastic mechanism underlying sleep-dependent consolidation, which has been most prominently studied at the glutamatergic synapse (Malenka and Bear, 2004). In this model, the postsynaptic membrane is depolarized above threshold by excitatory inputs that add up across time and space via AMPA receptors, thereby releasing the magnesium block of the NMDA receptor thus allowing for calcium influx and, downstream, for strengthening of the synapse, eg, by including further AMPA receptors into the postsynaptic membrane (Malenka and Nicoll, 1999). Blocking of both NMDA and AMPA receptors disturbs encoding of information, but only AMPA blockade impairs retrieval (Bast et al, 2005; Day et al, 2003). Conversely, enhancing NMDA receptor activation by administration of D-cycloserine (DCS), ie, a coagonist at the glycine binding site of the receptor, benefited declarative memory encoding (Onur et al, 2010). Yet, the role of glutamatergic neurotransmission for sleep-dependent offline consolidation of memories has been scarcely examined. In the developing cortex of cats, sleep-dependent ocular dominance plasticity was inhibited after blocking NMDA receptors (Aton et al, 2009). In adult humans, sleep-dependent consolidation of visual texture discrimination skill is deteriorated by the non-competitive NMDA receptor blocker ketamine or the competitive AMPA receptor blocker caroverine (Gais et al, 2008). However, these findings pertain to non-declarative types of memory not essentially relying on hippocampal networks.

Here, we tested contributions of glutamatergic neurotransmission to sleep-dependent consolidation of hippocampus-dependent declarative memory. As sleep-dependent consolidation of these memories is caused by the reactivation of firing patterns during SWS in neuron assemblies likely comprising glutamatergic activation, we expected that consolidation would be sensitive to blocking or enhancing glutamatergic neurotransmission during retention sleep. First, we investigated the effects of blocking AMPA receptors (by caroverine) or NMDA receptors (by ketamine) during retention sleep. Then we tested the effects of enhancing NMDA receptor function by post-learning administration of DCS.

\section{METHODS}

\section{Participants}

Altogether, 58 participants completed the study (caroverine: $n=15$, ketamine: $n=13$, DCS: $n=30$; see Supplementary Methods for details of the methods followed). Participants were healthy, nonsmoking, native German-speaking men (18-30 years). The experiments were approved by the ethics committee of the University of Luebeck. Written informed consent was obtained from all participants before participation. One participant revoked his consent after data acquisition in the DCS experiment, and his data were deleted.

\section{Design and Procedures}

Each of the experiments followed a randomized, doubleblind, placebo-controlled, within-subject, crossover design. In the DCS study, two different groups were recruited to compare the effects of DCS ( $v s$ placebo) during retention intervals of sleep $(n=16)$ and wakefulness $(n=14)$, respectively. Participants took part in two experimental sessions scheduled at least 14 days apart. Both sessions were identical, but for the administration of placebo or substance (caroverine: Calmaverine (Taphlan, Switzerland), intravenously, $16 \mathrm{mg} / \mathrm{h}$, corresponding to a total dose of $40 \mathrm{mg}$; plasma halftime: $25 \mathrm{~min}$, ketamine: Ketanest-S (Pfizer, USA), intravenously, $0.1 \mathrm{mg} / \mathrm{kg} / \mathrm{h}$, corresponding to a total dose of $0.25 \mathrm{mg} / \mathrm{kg}$; plasma halftime: $10-15 \mathrm{~min}$, DCS: Cycloserine Capsules (The Chao Center for Industrial Pharmacy and Contract Manufacturing, USA), $175 \mathrm{mg}$; plasma halftime: $10 \mathrm{~h}$, plasma maximum: 1-2 h; Figure 1 summarizes study designs). The administration of placebo and substance was performed in balanced order, ie, half of the sample received first placebo and then the active agent, for the other half the order of substance administration was reversed.

On experimental nights, the participants arrived at the sleep lab at 2000 hours and received a venous catheter for blood sampling. Following preparations for EEG and polysomnographical recordings, participants in all experiments learned declarative word-pair associations. In the DCS experiments, participants additionally learned emotional and neutral pictures and a procedural task, ie, finger sequence tapping. Learning took place always between 2100 and 2230 hours, and participants were asked to refrain from active rehearsal during the retention interval. Afterwards, in the caroverine and ketamine experiments participants received a second catheter for intravenous substance infusion. Infusions of ketamine and caroverine and respective placebo infusions (saline solution) started immediately after the first signs of sleep stage 2 and lasted for $2.5 \mathrm{~h}$. Participants were woken up $3 \mathrm{~h}$ after sleep onset (ie, the transition from sleep stage 1 to sleep stage 2) and retrieval was tested $30 \mathrm{~min}$ later. A retention interval of $3 \mathrm{~h}$ during the first half of the night was chosen, to restrict the effects of the substances to early nocturnal SWS-rich sleep, which has been shown to be the phase for reactivation of declarative memory (Rasch et al, 2007; Rudoy et al, 2009).

DCS and placebo were administered orally immediately before lights were put out (2300 hours), and participants were woken after $7.5 \mathrm{~h}$. This longer sleep period was chosen to ameliorate potential sleep-deprivation effects of reducing sleep to $3 \mathrm{~h}$ as, due to the substantially longer half-life of DCS, retrieval was shifted to the evening, ie, a time when plasma levels of DCS were negligible (Zhu et al, 2001). After the sleep period, participants then left the lab for approximately $12 \mathrm{~h}$ (during this time participants engaged in their usual activities) and returned for retrieval assessment. The wake control group of the DCS study was subjected to an identical protocol, but the beginning of the session was shifted by $10 \mathrm{~h}$ (arrival: 1000 hours, learning: 1100 hours) to ameliorate the effects of prolonged wakefulness; participants remained in the lab for the whole retention interval to prevent unintentional sleep.

\section{Polysomnography, Sleep Analysis, and EEG Power Analysis}

Sleep architecture was determined according to the standard polysomnographic criteria using EEG recordings from C3 and C4 (Rechtschaffen and Kales, 1968, details provided in the Supplementary Methods). For each night, 
total sleep time, ie, the time between the first detection of transition from sleep stage 1 to 2 and lights on and time spent in the different sleep stages, ie, wake; sleep stages 1,2, 3 , 4; SWS (defined by the sum of sleep stage 3 and 4) and rapid eye movement (REM) sleep was calculated in minutes.

Average power spectra were calculated at $\mathrm{Cz}$ for all sleep epochs of SWS and sleep stage 2 individually for the first and the second half of the night. Power spectra were calculated by Fast Fourier Transformations with a Hanning

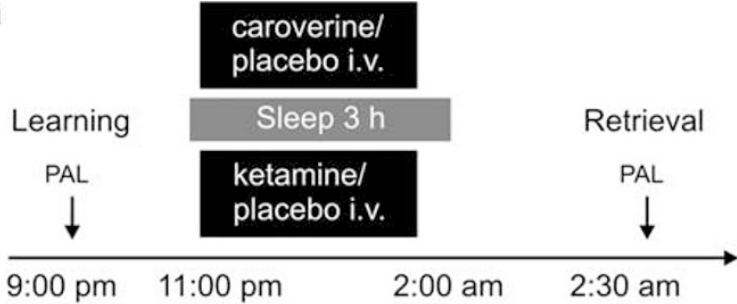

Learning DCS/

Retrieval

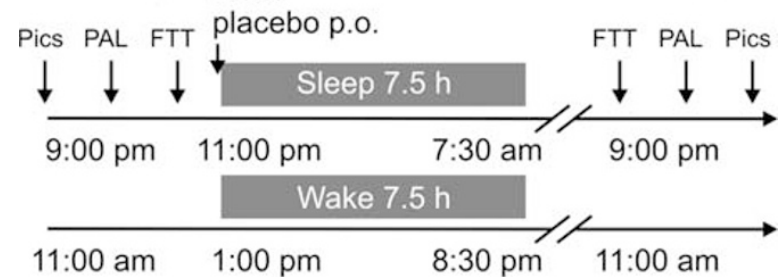

b Word Pairs
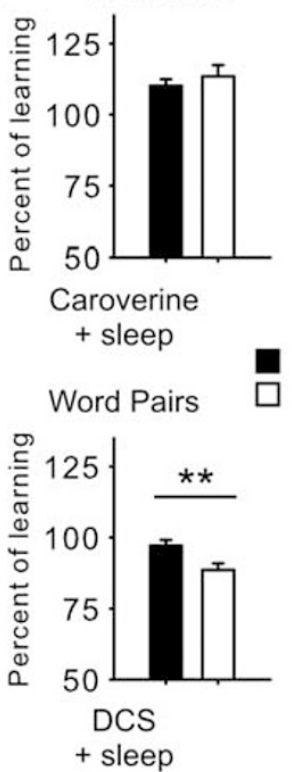

Finger tapping

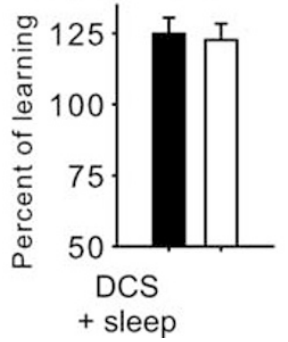

Substance Placebo
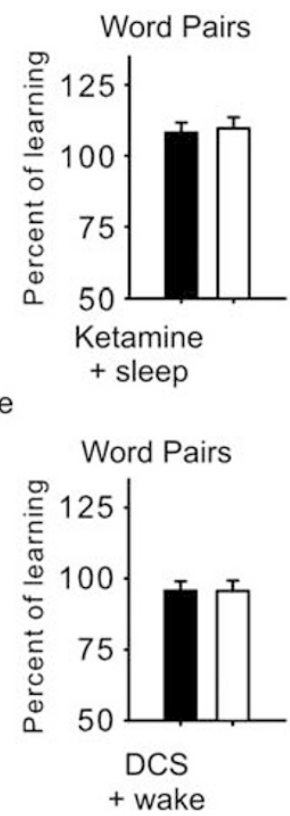

Finger tapping

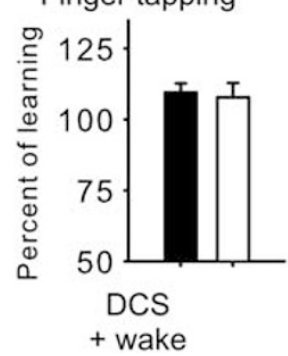

window applied to subsequent blocks of 2048 data points ( $\sim 10.24 \mathrm{~s}$, three blocks per 30-s epoch). Spectra were filtered by a five-point moving average to produce a smoothing of the FFT outcome. In the averaged spectra, mean power was determined for $0.5-1 \mathrm{~Hz}$ slow oscillation, $1-4 \mathrm{~Hz}$ delta, $9-12 \mathrm{~Hz}$ slow spindle, and the $12-15 \mathrm{~Hz}$ fast spindle frequency bands.

\section{Memory Tasks}

The declarative word-pair association task required learning a list of 40 word pairs. After presentation of the entire list, performance was tested using a cued-recall procedure, including feedback of the correct word. The cued-recall procedure was repeated until the participant reached a criterion of $60 \%$ correct responses. Retrieval at the end of the experimental session was tested using the same cuedrecall procedure as during the learning phase. Absolute differences between word pairs recalled at retrieval testing and on the criterion trial during learning served as a measure of overnight retention.

Additionally, in the DCS study one hundred emotional and neutral pictures (taken from the International Affective Picture System, Lang et al, 2008) were used to measure emotional memory consolidation. In the learning phase, 50 pictures of high arousal and negative valence were chosen for the emotional category and 50 pictures of low arousal and medium valence were chosen for the neutral category. Twenty-five pictures of each category were presented to the participants on a computer screen for $4 \mathrm{~s}$ each with an ISI of $1 \mathrm{~s}$. During retrieval testing at the end of the session, participants were asked to recall the pictures they had seen as accurately as possible and to record a description of each picture in writing. These descriptions were then compared with the pictures and correct answers were used as score for emotional memory performance.

Figure I (a) Study design: In the caroverine and ketamine studies, participants learned at 2100 hours and went to bed at 2300 hours. The retention interval was $3.5 \mathrm{~h}$, and half an hour after waking the participant, retrieval was tested at 0230 hours. In the D-Cycloserine (DCS) study, sleep condition participants also learned at 2100 hours and went to bed from 2300 hours to 0730 hours. The retention interval was $22 \mathrm{~h}$ and retrieval was tested at 2100 hours. In the wake condition, learning was shifted $10 \mathrm{~h}$ to II00 hours, and participants remained awake the whole retention interval until retrieval was tested at I I00 hours the next day. Approximate times of learning and retrieval are indicated, during learning criterion trials were the last cued recall during learning the word pairs and the last three blocks of learning the finger sequence. p.o.- oral administration, i.v.intravenous administration, PAL-word-pair association task, FTT-finger sequence tapping task, Pics - emotional and neutral pictures. (b) Overnight retention of word pairs and finger sequence tapping skills in the substance (black bars) and placebo condition (empty bars). Retention of word pairs is indicated by the mean $( \pm$ SEM) percentage of word pairs recalled at retrieval testing after the retention interval relative to recall performance on the criterion trial at learning before sleep (Please note that retention in the DCS experiments is generally lower than in the caroverine and ketamine experiments due to the longer retention interval). Overnight gains in finger sequence tapping are indicated by the mean $( \pm S E M)$ percentage of correctly tapped sequences per 30-s trial at retrieval testing relative to the average performance on the last three trials during training before the retention interval. $* * \leqslant \leqslant 0.01$, for pairwise comparisons between the effects of the treatments (caroverine: $n=15$, ketamine: $n=12$, DCS: $n=13$ for sleep condition, $n=14$ for wake condition). 
For procedural memory testing, in the DCS experiments, the finger sequence tapping task was adopted from earlier studies, indicating very robust sleep-dependent improvements on this task (Walker et al, 2003). It requires the participant to press repeatedly one of the two five-element sequences (eg, 4-1-3-2-4 or 4-2-3-1-4) with the fingers of the non-dominant hand on a keyboard as fast and as accurately as possible for 30-s epochs interrupted by 30 -s breaks. At learning, participants trained on 12 30-s trials, and during retrieval at the end of the session, particpants were tested on another three trials. Changes in performance across the sleep or wake-retention intervals were calculated as absolute differences in speed and error rate between the three trials at retrieval and the last three trials at learning. Effects unspecific to the actually learned sequence (eg, general increases in reaction time) were measured by assessing performance on three blocks of a new (untrained) sequence after recall of the trained sequence.

\section{Control Measures-Vigilance, Sleepiness, and Mood Ratings and Test of Encoding}

At the retrieval phase, vigilance, alertness, sleepiness, and mood were assessed using self-report measures, including the 'Eigenschaftswörterlist' (Janke and Debus, 1978), the Stanford sleepiness scale (SSS; Hoddes et al, 1973), and the Positive and Negative Affective Schedule (PANAS; Watson et al, 1988). In the DCS experiments, vigilance was additionally assessed by mean reaction times in a 5-min version of the psychomotor vigilance task (PVT; Dinges et al, 1997) that required pressing a button as fast as possible whenever a bright millisecond clock, present on a dark computer screen, started counting upward. After the button press, this clock displayed the reaction time. In these experiments, general capabilities of long-term memory retrieval were tested also using a word generation task. Participants had to generate as many words as possible starting with a certain letter $(\mathrm{P}$ or $\mathrm{M})$ or belonging to a defined category (hobby or profession) during a time of 2 min each.

Only in the wake control group of the DCS experiments, encoding (of a list of 16 three digit numbers) was measured. This measure was applied to test if DCS has an effect on encoding at high plasma concentrations during the wakeretention interval. At the end of a session, all participants were asked if they believed to have received an active agent or placebo.

\section{Analyses of Adrenocorticotropin (ACTH) and Cortisol}

Because blockers of glutamatergic transmission like ketamine can stimulate pituitary adrenal activity (Herman et al, 2004), we took blood samples once before and after learning as well as after retrieval. Additionally, blood samples were taken during the retention interval, ie, hourly during the first $4 \mathrm{~h}$ after substance intake and, in the DCS study, every $2 \mathrm{~h}$ during the second $4 \mathrm{~h}$. Sampling during the retention interval was performed via a long plastic tube from an adjacent room, leaving the participant to sleep undisturbed. Blood samples were immediately centrifuged and then stored at $-80^{\circ} \mathrm{C}$ until assay. Serum cortisol concentrations were assessed using the Immulite (Siemens Medical
Solutions Diagnostics, Los Angeles, CA; serum sensitivity, $0.2 \mu \mathrm{g} / \mathrm{dl}$, interassay coefficient of variation $<10 \%)$ ACTH was assessed in plasma (Immulite, Siemens Medical Solutions Diagnostics; sensitivity, $9 \mathrm{pg} / \mathrm{ml}$, interassay coefficient of variation $<9.6 \%$ ).

\section{Data Reduction and Statistical Analysis}

Data from one participant of the ketamine study were discarded due to $100 \%$ learning performance at baseline. Data from two participants of the DCS study were completely discarded because of poor sleep during the placebo night, and another participant's data were excluded from the finger sequence tapping task because his performance on the three criterion blocks was below than that during the first three blocks of training. Excluding these participants did not alter the reported effects. Data from one participant was excluded from the EEG power analysis due to artifacts in the data. Statistical analyses generally relied on analyses of variance (ANOVAs; SPSS version 21.0.0 for Windows), including a repeated measures factor 'treatment' (substance $v s$ placebo) and, where appropriate, the factor 'time point' (learning $v s$ retrieval). For analysis of pictures, an additional 'emotionality' factor was introduced, representing recall of neutral $v s$ emotional pictures. The analysis of EEG power during sleep included an additional factor for sleep stage (stage 2, SWS). Significant ANOVA interactions were specified by post-hoc $t$-tests. Degrees of freedom were corrected according to Greenhouse-Geisser where appropriate.

\section{RESULTS}

\section{Memory Tasks}

Neither caroverine nor ketamine significantly changed retention of word pairs in comparison with respective placebo treatments (all $p>0.53$, see Table 1 and Figure 1 for a summary of results). Learning performance also did not differ between the active agents and respective placebo conditions (all $t \leqslant 1.61, p \geqslant 0.13$ ).

DCS administration before the sleep-retention interval distinctly improved recall of word pairs at retrieval testing after the retention interval. This effect was confirmed by significance for the ANOVA treatment $\times$ time point interaction $\left(F_{(1,12)}=9.33, p \leqslant 0.01\right.$, Table 1 and Figure 1$)$. By contrast, DCS administered before a wake-retention interval did not improve word-pair retention $(p \geqslant 0.99)$. During learning, there were no evident differences between placebo and DCS conditions concerning amounts of learned word pairs and trials to criterion (all $t \leqslant 1.19, p \geqslant 0.19$ ). An ANOVA including both the sleep and wake groups of the DCS study (represented by an additional 'sleep/wake' factor) revealed a trend for the treatment $\times$ time point $\times$ sleep/wake interaction $\left(F_{(1,25)}=3.15, p=0.09\right)$.

The emotional memory task did not reveal any differences as measured by the amount of freely recalled emotional and neutral pictures between DCS and placebo conditions both in the sleep group and in the wake group of this study $(p \geqslant 0.29$, for respective main and interaction effects of treatment, Table 2). Independent of the treatment condition, generally more emotional than neutral pictures 
Table I Word-Pair Memory Task

\begin{tabular}{|c|c|c|c|}
\hline & Substance & Placebo & $p$ \\
\hline \multicolumn{4}{|l|}{ Caroverine + sleep } \\
\hline Blocks to criterion & $1.53 \pm 0.17$ & $1.60 \pm 0.16$ & NS \\
\hline Learning & $29.60 \pm 0.83$ & $28.13 \pm 0.84$ & NS \\
\hline Retrieval & $32.53 \pm 0.98$ & $31.67 \pm 0.98$ & NS \\
\hline Absolute difference & $2.93 \pm 0.64$ & $3.53 \pm 1.0$ & NS \\
\hline Percentage of learning & $110.14 \pm 2.27$ & | $13.43 \pm 4.00$ & NS \\
\hline \multicolumn{4}{|l|}{ Ketamine + sleep } \\
\hline Blocks to criterion & $1.50 \pm 0.15$ & $1.58 \pm 0.15$ & NS \\
\hline Learning & $29.19 \pm 0.98$ & $27.93 \pm 1.13$ & NS \\
\hline Retrieval & $31.35 \pm 0.91$ & $30.23 \pm 0.69$ & NS \\
\hline Absolute difference & $2.16 \pm 0.94$ & $2.29 \pm 0.93$ & NS \\
\hline Percentage of learning & $108.12 \pm 3.58$ & $109.65 \pm 3.90$ & NS \\
\hline \multicolumn{4}{|l|}{ DCS + sleep } \\
\hline Blocks to criterion & $1.85 \pm 0.15$ & $2.00 \pm 0.25$ & NS \\
\hline Learning & $27.85 \pm 0.83$ & $28.31 \pm 1.11$ & NS \\
\hline Retrieval & $27.00 \pm 0.87$ & $25.08 \pm 1.16$ & NS \\
\hline Absolute difference & $-0.85 \pm 0.55$ & $-3.23 \pm 0.59$ & **⿻丷木 \\
\hline Percentage of learning & $97.10 \pm 1.99$ & $88.56 \pm 2.27$ & *** \\
\hline \multicolumn{4}{|l|}{ DCS + wake } \\
\hline Blocks to criterion & $1.50 \pm 0.14$ & $1.71 \pm 0.19$ & NS \\
\hline Learning & $28.64 \pm 0.90$ & $29.21 \pm 0.82$ & NS \\
\hline Retrieval & $27.36 \pm 1.19$ & $27.93 \pm 1.32$ & NS \\
\hline Absolute difference & $-1.29 \pm 1.01$ & $-1.29 \pm 1.07$ & NS \\
\hline Percentage of learning & $95.78 \pm 3.34$ & $95.68 \pm 3.69$ & NS \\
\hline
\end{tabular}

Abbreviation: NS, not significant.

$* * * 0.01$.

Mean $( \pm$ SEM $)$ values are given for the active agent and placebo conditions. Total amount of recalled words is given for criterion trials at learning and at retrieval. Additionally, percentage of values of retrieved words are provided relative to learning performance (set to 100\%).

were remembered (sleep: $F_{(1,13)}=21.06$ and $p \leqslant 0.01$, wake: $\left.F_{(1,13)}=26.74, p \leqslant 0.01\right)$.

Procedural finger sequence tapping was not differentially affected by DCS or placebo. The overnight gains in tapping speed and accuracy were comparable in both conditions (all $p \geqslant 0.27$, Table 2), and this was also true for the wake control group (all $p \geqslant 0.16$ ). There was also no difference evident between DCS and placebo conditions at training or concerning performance on the untrained control sequence during the retrieval phase (all $p \geqslant 0.26$ ). The ANOVA including both the sleep and the wake condition of the DCS study revealed that the sleep group improved their performance more during the retention interval $\left(F_{(1,24)}=8.64, p \leqslant 0.01\right.$ for time point $\times$ sleep/wake $)$.

\section{Sleep}

Infusion of ketamine compared with placebo reduced the time spent in stage 2, SWS, and REM sleep and increased time in wakefulness (wake: $t_{(12)}=2.55, p \leqslant 0.05$, stage 2 : $t_{(12)}=-2.75, p \leqslant 0.05$; SWS: $t_{(12)}=-2.14, p \leqslant 0.05$, stage 4 : $\mathrm{t}_{(12)}=-2.15, \quad p \leqslant 0.05 ; \quad$ REM: $t_{(12)}=-2.50, \quad p \leqslant 0.05$, Table 3). Under the influence of caroverine, there was a trend towards less time spent in stage 4 sleep $\left(t_{(13)}=1.79\right.$, $p=0.10)$. Oral administration of DCS before sleep increased time in wakefulness $\left(t_{(12)}=2.66, p \leqslant 0.05\right)$ and stage 1 sleep $\left(t_{(12)}=2.27, \quad p \leqslant 0.05\right)$ and reduced REM sleep $\left(t_{(12)}=-3.51, p \leqslant 0.01\right.$; Table 3$)$. There was no evident correlation between DCS-induced changes in sleep architecture and improvements in the retention of word pairs (all $r \leqslant 0.34$ and $p \geqslant 0.26$, changes were calculated individually with reference to the placebo condition).

More fine-grained analyses of EEG power spectra at $\mathrm{Cz}$ revealed a power reduction around the spindle maximum during stage 2 sleep following DCS (Figure 2). ANOVA on the fast spindle band $(12-15 \mathrm{~Hz})$ confirmed significance for the sleep stage $\times$ treatment interaction $\left(F_{(1,11)}=9.47\right.$, $p \leqslant 0.01 ; t_{(11)}=2.71, \quad p \leqslant 0.05$ for post-hoc comparison between the treatments for stage 2 spindle power). The reducing effect of DCS on stage 2 sleep spindle power appeared to be less pronounced during the first than the second half of sleep $\left(t_{(11)}=-2.95, p \leqslant 0.01\right.$, for pairwise comparison between the effects of treatment, $F_{(1,11)}=7.87$, $p \leqslant 0.05$, for treatment $\times$ night half). However, this was due to the fact that during the first half DCS simultaneously enhanced beta power, with this effect extending into the upper $(>14-\mathrm{Hz})$ range of fast spindle frequencies (Figure 2). Analyses on the other bands did not show any significant effects of treatment $(p \geqslant 0.16)$. There was no correlation between DCS-induced changes in the spindle band and differences in the retention of word pairs (all $r \leqslant 0.26$ and $p \geqslant 0.39$ ).

\section{Control Measures}

In the caroverine study, we found no differences between the treatments in subjective measures of vigilance, alertness, sleepiness, or mood during the retrieval phase $(p \geqslant 0.19)$. These measures also did not differ between treatments in the ketamine study $(p \geqslant 0.58)$; however, three participants reported slight nausea after awakening on the ketamine nights. Cortisol and ACTH levels were not differentially affected by caroverine or placebo $(p \geqslant 0.31)$. Under ketamine, cortisol was increased at the end of the infusion (between 0100 and 0200 hours; ketamine: $4.54 \pm 3.67 \mu \mathrm{g} / \mathrm{dl}$; placebo $2.80 \pm 1.96 \mu \mathrm{g} / \mathrm{dl} ; p \leqslant 0.05)$ and ACTH concentrations showed a corresponding trend $(p=0.10)$. A positive relation between differences in cortisol level and differences in word-pair retention in the sleep condition was found (0200 hours: $r=0.62$ and $p \leqslant 0.05$ ); however, it did not survive multiple comparison correction.

Subjective measures of vigilance, sleepiness, and mood, as well as behavioral measures of vigilance and general retrieval capabilities at retrieval all remained unaffected after administration of DCS $(p \geqslant 0.11)$. An additional control test performed in the wake control group of the DCS study, which tested if DCS influences encoding during wakefulness, revealed that $4 \mathrm{~h}$ after DCS intake intrusions (from previous testing immediately after and $2 \mathrm{~h}$ after substance intake) were reduced (DCS: $0.21 \pm 0.11$; placebo: $0.93 \pm 0.27, t_{(13)}=-2.92, p \leqslant 0.01, F_{(1,13)}=8.29, p \leqslant 0.01$ for treatment $\times$ time point). Levels of cortisol and ACTH did not differ between treatment conditions $(p \geqslant 0.26)$.

Participants could differentiate ketamine and placebo $\left(X^{2}{ }_{(1)}=22.29, p \leqslant 0.01\right)$. However, caroverine and placebo as well as DCS and placebo could not be discriminated $(p \geqslant 0.25)$. 
Table 2 Emotional and Procedural Memory

\begin{tabular}{|c|c|c|c|}
\hline & DCS & Placebo & $p$ \\
\hline \multicolumn{4}{|l|}{ Emotional and neutral pictures } \\
\hline \multicolumn{4}{|l|}{ Sleep } \\
\hline Emotional & $7.54 \pm 0.83$ & $8.23 \pm 0.74$ & NS \\
\hline Neutral & $4.77 \pm 0.86$ & $4.85 \pm 0.85$ & NS \\
\hline Total & $12.31 \pm 1.29$ & $13.08 \pm 1.36$ & NS \\
\hline \multicolumn{4}{|l|}{ Wake } \\
\hline Emotional & $6.14 \pm 0.72$ & $6.79 \pm 0.88$ & NS \\
\hline Neutral & $4.00 \pm 0.60$ & $4.57 \pm 0.60$ & NS \\
\hline Total & $10.14 \pm 1.08$ & $11.36 \pm 1.34$ & NS \\
\hline \multicolumn{4}{|l|}{ Finger sequence tapping } \\
\hline \multicolumn{4}{|l|}{ Sleep } \\
\hline Learning & $17.11 \pm 1.18$ & $16.36 \pm 1.21$ & NS \\
\hline Retrieval & $21.03 \pm 1.26$ & $19.75 \pm 1.26$ & NS \\
\hline Absolute difference & $3.91 \pm 0.72$ & $3.39 \pm 0.89$ & NS \\
\hline Percentage of learning & $124.87 \pm 5.69$ & $122.66 \pm 5.75$ & NS \\
\hline Untrained sequence & $13.50 \pm 1.55$ & $13.47 \pm 0.83$ & NS \\
\hline \multicolumn{4}{|l|}{ Wake } \\
\hline Learning & $18.83 \pm 1.00$ & $18.78 \pm 1.00$ & NS \\
\hline Retrieval & $20.60 \pm 1.21$ & $20.10 \pm 1.29$ & NS \\
\hline Absolute difference & $1.76 \pm 0.54$ & $1.31 \pm 0.93$ & NS \\
\hline Percentage of learning & $109.56 \pm 3.18$ & $107.86 \pm 5.04$ & NS \\
\hline Untrained sequence & $12.86 \pm 0.72$ & $12.66 \pm 0.72$ & NS \\
\hline
\end{tabular}

Abbreviation: NS, not significant.

*** $p \leqslant 0.01, * p \leqslant 0.05$.

Mean $( \pm$ SEM $)$ values are given for the DCS and placebo condition. Top:

number of correctly remembered emotional, neutral, and of total pictures in the emotional memory task. Bottom: average number of correctly tapped

sequences for the finger sequence tapping during the last three 30-s trials at learning, the three trials at retrieval and for the untrained sequence at retrieval. Additionally, percentage of values of correctly tapped sequences at retrieval are provided relative to learning performance (set to 100\%).

\section{DISCUSSION}

Evidence from animal and human studies indicates that the consolidation of hippocampus-dependent declarative memory relies on the reactivation of newly encoded neuronal representations during post-learning SWS (Diekelmann and Born, 2010; Rasch et al, 2007; Ribeiro et al, 2007; Wilson and McNaughton, 1994). During SWS, the same sequential firing patterns are observed in hippocampal neuron assemblies as during encoding before sleep (O'Neill et al, 2010). These reactivations that typically coincide with sharp wave-ripples in the hippocampal EEG are thought to involve excitatory glutamatergic synapses (Behrens et al, 2005; Dupret et al, 2010; King et al, 1999). Repeated reactivations are hence expected to induce plastic synaptic changes within these hippocampal assemblies and in extrahippocampal output structures that generally contribute to the strengthening of respective memory representations. A candidate mechanism mediating memory consolidation in this context is glutamatergic LTP, where the AMPA receptor is responsible for the fast signal transfer and the NMDA receptor acts as coincidence detector for the induction of LTP (Malenka and Nicoll, 1999). Consequently, here we found that facilitating the response of the NMDA receptor to glutamate with the coagonist DCS improved the consolidation of hippocampus-dependent word-pair memories during sleep. Yet, contrary to our expectation, the straightforward approach of blocking AMPA or NMDA receptors did not impair declarative memory consolidation. This pattern suggests that effective hippocampal memory replay during sleep does not simply rely on the reactivation of synaptic AMPA and NMDA receptors that contributed to encoding, thereby suggesting that processes other than classical glutamatergic LTP essentially contribute to sleep-dependent declarative memory consolidation.

The improvement in word-pair recall after DCS administration before the retention period of sleep can easily be explained by the coagonistic effect of DCS-enhancing activity of the NMDA receptor and, consequently, enhancing LTP after assembly reactivation in hippocampal networks. Whether the DCS effect reflects plastic changes at glutamatergic synapses within and/or outside the hippocampus cannot be inferred from the present data. Observations that retrieval of word-pair memories within 2 days after retention sleep was associated with an increase in activity primarily within the hippocampus (Gais et al, 2007) suggest that the immediate effects of DCS on the first night after learning strengthen the memory trace within the hippocampus itself. Also arguing in favor of this, DCS reduced power of the fast sleep spindles, which have been considered a mechanism supporting the transfer of reactivated memory information to extrahippocampal sites (Inostroza and Born, 2013; Mölle and Born, 2011; Siapas and Wilson, 1998). However, it is also possible that the positive effect of DCS on the representation outweighs the negative impact of reduced sleep spindles.

Notably, DCS did not influence retention of word pairs when given before a wake interval. Assuming spontaneous reactivations also occurred during wake-retention intervals, this finding supports the concept that reactivations during sleep serve different functions from reactivations during wakefulness (Diekelmann et al, 2011). Indeed during wakefulness, reactivations are thought to exert twofold functions, ie, to transiently labilize the representation and to support re-encoding of the stimulus (Hardt et al, 2013; Nader and Hardt, 2009), and the same reactivation of memories by odor cues during sleep that facilitated memory, when applied during wake, led to decreased declarative memory retention (Diekelmann et al, 2011). Missing interference together with the instruction not to rehearse the learned tasks may have led to DCS not changing memory in any direction during the wake-retention interval, as could have been expected. As to encoding of hippocampal memories, improving effects of DCS have been revealed in most (eg, Kuriyama et al, 2011c; Lee et al, 2006; Onur et al, 2010) but not in all studies (eg, Kuriyama et al, 2011a), and an improving effect of DCS on encoding also fits our findings of reduced intrusions at immediate recall of numbers after DCS administration in the wake condition-although, the effect was not marked. Thus, speculating that DCS has a similar benefiting effect on re-encoding, the missing effect of DCS on retention of hippocampal memories across wake intervals might point towards a parallel enhancing effect of DCS on the reactivation-induced labilization of memories (Ben Mamou et al, 2006; Lee et al, 2006).

DCS did not enhance overnight gains in procedural finger sequence tapping skills and also failed to affect memory 
Table 3 Sleep Parameters

\begin{tabular}{|c|c|c|c|}
\hline & Substance & Placebo & $p$ \\
\hline \multicolumn{4}{|l|}{ Caroverine } \\
\hline Wakefulness & $2.80 \pm 1.21$ & $2.41 \pm 0.60$ & NS \\
\hline Stage 1 & $22.29 \pm 4.59$ & $29.5 \pm 4.78$ & NS \\
\hline Stage 2 & $96.18 \pm 5.63$ & $87.57 \pm 7.65$ & NS \\
\hline Stage 3 & $44.61 \pm 4.39$ & $42.14 \pm 3.35$ & NS \\
\hline Stage 4 & $5.46 \pm 1.64$ & $8.07 \pm 2.11$ & $t$ \\
\hline REM & $|1.67 \pm 2.5|$ & $9.96 \pm 3.32$ & NS \\
\hline SWS & $50.07 \pm 5.18$ & $50.21 \pm 4.49$ & NS \\
\hline TST & $184.28 \pm 2.05$ & $182.07 \pm 3.36$ & NS \\
\hline \multicolumn{4}{|l|}{ Ketamine } \\
\hline Wakefulness & $47.96 \pm 13.87$ & $14.69 \pm 4.29$ & $*$ \\
\hline Stage 1 & $26.38 \pm 4.49$ & $20.00 \pm 2.76$ & NS \\
\hline Stage 2 & $77.69 \pm 9.32$ & $104.12 \pm 5.05$ & * \\
\hline Stage 3 & $|9.50 \pm 4.6|$ & $23.92 \pm 3.25$ & NS \\
\hline Stage 4 & $2.58 \pm 1.18$ & $6.08 \pm 1.96$ & * \\
\hline REM & $|2.62 \pm 3.6|$ & $22.96 \pm 4.17$ & $*$ \\
\hline SWS & $22.08 \pm 5.53$ & $30.00 \pm 4.35$ & $*$ \\
\hline TST & $186.73 \pm 1.73$ & $191.77 \pm 5.5$ & NS \\
\hline \multicolumn{4}{|l|}{ DCS } \\
\hline Wakefulness & $19.54 \pm 3.43$ & $9.84 \pm 2.62$ & $*$ \\
\hline Stage 1 & $31.27 \pm 2.89$ & $25.30 \pm 2.03$ & * \\
\hline Stage 2 & $213.77 \pm 8.88$ & $215.69 \pm 10.52$ & NS \\
\hline Stage 3 & $60.46 \pm 6.37$ & $56.69 \pm 6.59$ & NS \\
\hline Stage 4 & $32.96 \pm 7.61$ & $33.19 \pm 7.77$ & NS \\
\hline REM & $83.73 \pm 5.39$ & $101.85 \pm 6.95$ & **⿻丷木 \\
\hline SWS & $93.42 \pm 10.07$ & $89.88 \pm 10.47$ & NS \\
\hline TST & $445.38 \pm 3.18$ & $447.08 \pm 3.76$ & NS \\
\hline
\end{tabular}

Abbreviation: NS, not significant.

*** $p \leqslant 0.01, * p \leqslant 0.05, t \leqslant 0.10$.

$\mathrm{REM}=$ rapid eye movement sleep; SWS = slow wave sleep; TST = total sleep time.

Mean $( \pm$ SEM $)$ values of minutes spent in the different sleep stages are given for the active agent and placebo conditions.

after sleep for emotional and neutral pictures, which might reflect that sleep-associated consolidation of these memories is not primarily a consequence of hippocampal reactivation (Debas et al, 2010; Diekelmann and Born, 2010; Karni et al, 1998; Wagner et al, 2001 but see also Albouy et al, 2008). The findings concur with previous studies that likewise failed to observe DCS-induced changes in overnight gains in cognitive skill, although benefits in retention occurred for working memory training and emotional memories when participants were awake (Kalisch et al, 2009; Kuriyama et al, 2011b). Interestingly, in a previous study of ours both blocking of NMDA receptors and of AMPA receptors impaired sleep-dependent gains in a procedural visual texture discrimination task (Gais et al, 2008). Indeed, against this backdrop, the present pattern of a selective enhancing influence of DCS on sleepassociated declarative memory consolidation in the absence of changes in overnight benefits for procedural skills or recognition memories supports the view that DCS specifically acts on hippocampal memory reactivations during sleep, as sleep-associated gains in those memories are less dependent on such reactivations.

Paradoxically, whereas the NMDA receptor coagonist DCS significantly enhanced hippocampus-dependent mem- ory consolidation of word-pair associations during sleep, the consolidation process remained entirely unaffected after disrupting glutamatergic neurotransmission by administration of either the NMDA receptor antagonist ketamine or the AMPA receptor antagonist caroverine. It is unlikely that the missing effect after ketamine or caroverine is due to a too low dosing of the substances, because in a previous study of ours (Gais et al, 2008) infusion of the substances at very similar concentrations (in case of caroverine even slightly lower concentrations were used in that study) effectively blocked sleep-dependent consolidation of visual texture discrimination skills. Still, it could be argued that the duration of administration matters, as in that study substances were infused for a longer $(6 \mathrm{~h})$ interval, whereas here we restricted administration to a 2.5 -h interval in which neuronal reactivations of memories are thought to preferentially occur in hippocampal networks. Although a longer infusion duration cannot be entirely excluded as a prerequisite for the substances to become effective at hippocampal sites, increased levels of cortisol and reports of side effects like nausea observed after ketamine confirmed central nervous efficacy of the substance even with the shorter infusion interval. Studies on guinea pigs and using PET imaging in humans indicate that both ketamine and caroverine quickly reach central nervous sites of action within minutes after intravenous administration (Chen et al, 2003; Hartvig et al, 1995). Also, power analyses did not provide any hint that putative blocking effects of ketamine and caroverine on consolidation of hippocampal memories during sleep were just not strong enough to be revealed with the limited sample size of our study. Since the effect size for DCS improving word-pair memory retention during sleep was large $(d=0.85$; Cohen, 1992), and power analysis (using $G^{*}$ Power; Faul et al, 2007) with $(1-\beta)$ set to 0.80 led to unreasonably large sample sizes of $n=423$ and $n=6808$ to reach significance $(\alpha=0.05)$ for the differences reported in the caroverine and ketamine conditions. Another factor to be considered in this context is that the ketamine and caroverine studies differed from the DCS study with respect to the duration of sleep, which in the DCS study covered the whole night due to its longer plasma half-life and the resulting longer retention interval; however, its plasma maximum falls into the first half of the night. Nevertheless, numerous studies have consistently shown that restricting manipulations to a 3 -h period of early nocturnal SWS-rich sleep can effectively change consolidation of declarative memory (eg, Barrett and Ekstrand, 1972; Marshall et al, 2004; Plihal and Born, 1997), excluding the amount of sleep per se as factor preventing effects of ketamine or caroverine. Still, although previous work has demonstrated that sleep-dependent consolidation of our word-pair task involved the hippocampus (Gais et al, 2007), not investigating other hippocampusdependent forms of memory, eg, object location, sequence, or episodic memory, or other doses of the substances limits the explanatory power of our findings.

Although any explanation of these findings remains tentative, the data imply that efficacy of hippocampal memory reactivation during sleep might not rely exclusively on activation mediated by AMPA and NMDA receptors. Reactivations during wakefulness associated with memory retrieval do not appear to rely on activation of NMDA 

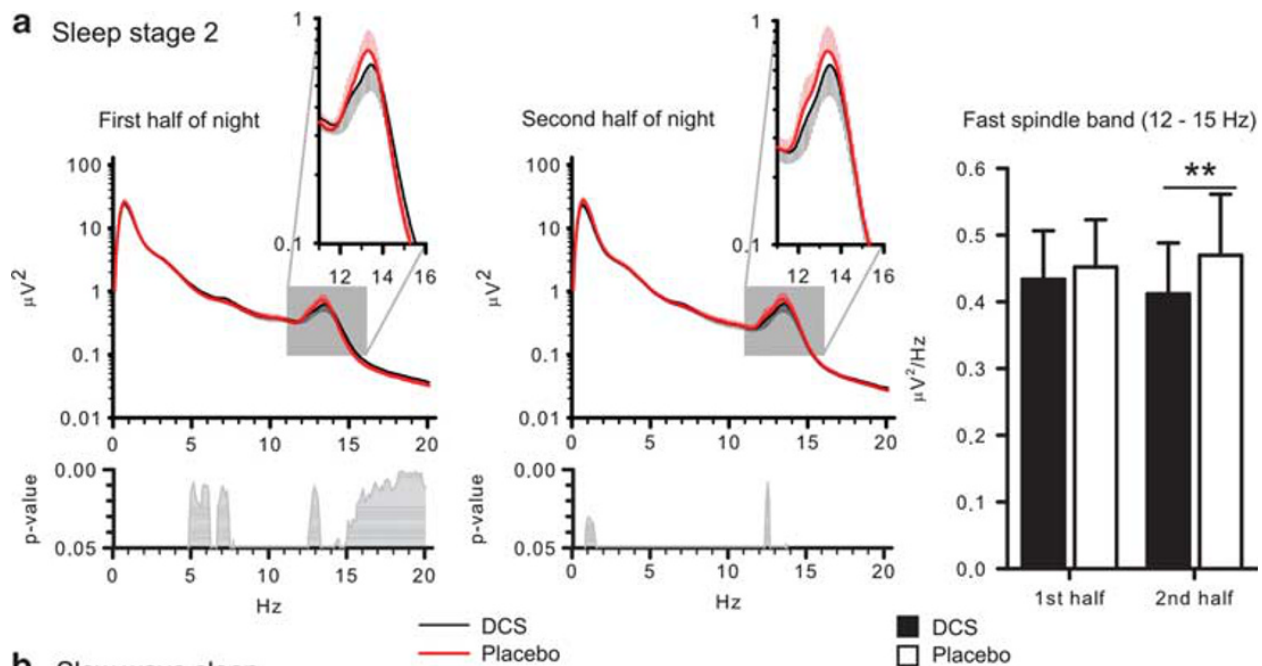

b Slow wave sleep
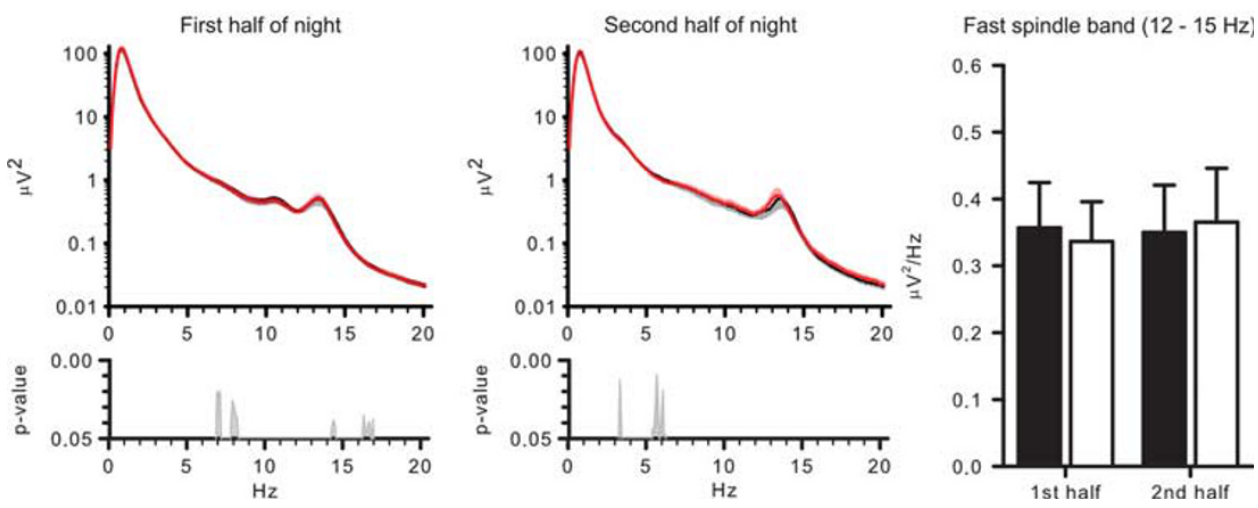

Figure 2 Mean EEG power ( \pm SEM) during sleep stage 2 (a) and slow wave sleep (b) between $0.1-20 \mathrm{~Hz}$ in the first half of night (left panels) and second half of night (middle panels). Respective bottom panels indicate $p$-values for pairwise comparisons between the treatment conditions (placebo-thick red line, D-Cycloserine (DCS) - thin black line). Right panels show mean ( \pm SEM) power in the fast spindle $(12-15 \mathrm{~Hz})$ band during the first and the second night half (Please note that only differences were considered meaningful that remained for the mean power of the entire frequency band of interest). $* * 0 \leqslant 0.0 \mathrm{I}$, for pairwise comparisons between the effects of the treatments $(n=12)$.

receptors anyway, although they involve AMPA receptor activation (Bast et al, 2005; Day et al, 2003). Moreover, ketamine-induced blockade of NMDA receptors can be ameliorated by activating metabotropic glutamate receptor 5 (mGluR5; Chen et al, 2011). Thus, in synapses potentiated during encoding, glutamatergic signalling might shift to mGluR5, making them insensitive to ketamine blockade, although, such a shift itself would not explain why synaptic efficacy can still be enhanced via DCS.

A more plausible explanation may be derived from work indicating that activation of hippocampal NMDA receptors, and possibly also AMPA receptors, are not only responsible for LTP induction but are also involved in subsequent depotentiation, ie, LTD, thereby mediating forgetting (Rosenzweig et al, 2002; Villarreal et al, 2002). In the hippocampus, LTP is preferentially mediated by NMDA receptors containing the NR2A subunit, whereas LTD is mediated by NR2B containing receptors (Liu et al, 2004). Furthermore, there is evidence that DCS preferentially acts via NR2A containing receptors to enhance LTP (Billard and Rouaud, 2007; Kochlamazashvili et al, 2012; Sheinin et al, 2001), whereas ketamine provides an unspecific blockade of both these NMDA receptor subtypes. These findings suggest the following scenario for the present experiments: Encod- ing of word pairs leads to the potentiation of specific hippocampal assemblies, which is accompanied by a selective upregulation of NMDA receptors containing the NR2A subunit (Baez et al, 2013). Glutamatergic reactivation of these assemblies during sleep preferentially enhances NR2A-mediated LTP, whereas NR2B-mediated LTD prevails in networks not specifically potentiated during wakefulness. Ketamine (and caroverine) leave the reactivation-dependent memory enhancement during sleep unaffected as the proportional activation of LTP and LTD inducing NMDA receptors remains unchanged. By contrast, DCS by preferentially activating NR2A containing receptors strengthens LTP and thus enhances the consolidating effect of sleep on hippocampal memories. This view is very much in line with a recently proposed account on the role of sleep in active decay processes and forgetting (Hardt et al, 2013). Following this view, a factor mediating the effect of sleepinduced LTP and LTD on retention may be represented by the protein kinase $\mathrm{C}$ isoform $\mathrm{M}$-zeta $(\operatorname{PKM} \zeta)$, which has been shown to sustain hippocampal memories by regulating AMPA receptor trafficking to the active zone of the synapse and might simultaneously regulate forgetting of such memories as LTD has been shown to degrade $\mathrm{PKM} \zeta$ (Hardt et al, 2010; Hrabetova and Sacktor, 2001; Migues 
et al, 2010). Together with the present research, this account offers a mechanism for an active process of synaptic consolidation working in balance with processes of synaptic downscaling to sustain hippocampus function and long-term memory (Born and Feld, 2012; Diekelmann and Born, 2010; Tononi and Cirelli, 2006).

This scenario relating glutamatergic signalling and reactivation in hippocampal neuron assemblies to balanced processes of memory consolidation and forgetting is clearly in need of further experimentation but would plausibly reconcile findings of SWS being simultaneously involved in freeing of capacity for new learning (Antonenko et al, 2013; Van Der Werf et al, 2009) and memory consolidation (Diekelmann and Born, 2010; Marshall et al, 2006).

\section{FUNDING AND DISCLOSURE}

The authors declare no conflict of interests.

\section{ACKNOWLEDGEMENTS}

We thank Felix Machleidt for medical supervision of the study; Simon Schütt, Cornelia Herzmann, and Maike Hansmann for assisting in data collection; and Martina Grohs and Heidi Ruf for performing blood analyses. This research was supported by a grant from the Deutsche Forschungsgemeinschaft SFB 654 'Plasticity and Sleep'.

\section{REFERENCES}

Albouy G, Sterpenich V, Balteau E, Vandewalle G, Desseilles M, Dang-Vu T et al (2008). Both the hippocampus and striatum are involved in consolidation of motor sequence memory. Neuron 58: $261-272$.

Antonenko D, Diekelmann S, Olsen C, Born J, Molle M (2013). Napping to renew learning capacity: enhanced encoding after stimulation of sleep slow oscillations. Eur J Neurosci 37: 1142-1151.

Aton SJ, Seibt J, Dumoulin M, Jha SK, Steinmetz N, Coleman T et al (2009). Mechanisms of sleep-dependent consolidation of cortical plasticity. Neuron 61: 454-466.

Baez MV, Oberholzer MV, Cercato MC, Snitcofsky M, Aguirre AI, Jerusalinsky DA (2013). NMDA receptor subunits in the adult rat hippocampus undergo similar changes after 5 minutes in an open field and after LTP induction. PLoS One 8: e55244.

Barrett TR, Ekstrand BR (1972). Effect of sleep on memory. 3. Controlling for time-of-day effects. J Exp Psychol 96: 321-327.

Bast T, da Silva BM, Morris RG (2005). Distinct contributions of hippocampal NMDA and AMPA receptors to encoding and retrieval of one-trial place memory. J Neurosci 25: 5845-5856.

Behrens CJ, van den Boom LP, de Hoz L, Friedman A, Heinemann U (2005). Induction of sharp wave-ripple complexes in vitro and reorganization of hippocampal networks. Nat Neurosci 8: 1560-1567.

Ben Mamou C, Gamache K, Nader K (2006). NMDA receptors are critical for unleashing consolidated auditory fear memories. Nat Neurosci 9: 1237-1239.

Billard JM, Rouaud E (2007). Deficit of NMDA receptor activation in CA1 hippocampal area of aged rats is rescued by D-cycloserine. Eur J Neurosci 25: 2260-2268.

Born J, Feld GB (2012). Sleep to upscale, sleep to downscale: balancing homeostasis and plasticity. Neuron 75: 933-935.

Chauvette S, Seigneur J, Timofeev I (2012). Sleep oscillations in the thalamocortical system induce long-term neuronal plasticity. Neuron 75: 1105-1113.
Chen HH, Liao PF, Chan MH (2011). mGluR5 positive modulators both potentiate activation and restore inhibition in NMDA receptors by PKC dependent pathway. J Biomed Sci 18: 19.

Chen Z, Duan M, Lee H, Ruan R, Ulfendahl M (2003). Pharmacokinetics of caroverine in the inner ear and its effects on cochlear function after systemic and local administrations in guinea pigs. Audiol Neurootol 8: 49-56.

Cohen J (1992). A power primer. Psychol Bull 112: 155-159.

Csicsvari J, Hirase H, Czurko A, Mamiya A, Buzsaki G (1999). Fast network oscillations in the hippocampal CA1 region of the behaving rat. J Neurosci 19: RC20.

Day M, Langston R, Morris RG (2003). Glutamate-receptormediated encoding and retrieval of paired-associate learning. Nature 424: 205-209.

Debas K, Carrier J, Orban P, Barakat M, Lungu O, Vandewalle G et al (2010). Brain plasticity related to the consolidation of motor sequence learning and motor adaptation. Proc Natl Acad Sci USA 107: 17839-17844.

Diekelmann S, Born J (2010). The memory function of sleep. Nat Rev Neurosci 11: 114-126.

Diekelmann S, Buchel C, Born J, Rasch B (2011). Labile or stable: opposing consequences for memory when reactivated during waking and sleep. Nat Neurosci 14: 381-386.

Dinges DF, Pack F, Williams K, Gillen KA, Powell JW, Ott GE et al (1997). Cumulative sleepiness, mood disturbance, and psychomotor vigilance performance decrements during a week of sleep restricted to 4-5 hours per night. Sleep 20: 267-277.

Dupret D, O’Neill J, Pleydell-Bouverie B, Csicsvari J (2010). The reorganization and reactivation of hippocampal maps predict spatial memory performance. Nat Neurosci 13: 995-1002.

Espinosa F, Kavalali ET (2009). NMDA receptor activation by spontaneous glutamatergic neurotransmission. J Neurophysiol 101: 2290-2296.

Faul F, Erdfelder E, Lang AG, Buchner A (2007). G*Power 3: a flexible statistical power analysis program for the social, behavioral, and biomedical sciences. Behav Res Methods 39: 175-191.

Frank MG, Benington JH (2006). The role of sleep in memory consolidation and brain plasticity: dream or reality? Neuroscientist 12: 477-488.

Gais S, Albouy G, Boly M, Dang-Vu TT, Darsaud A, Desseilles M et al (2007). Sleep transforms the cerebral trace of declarative memories. Proc Natl Acad Sci USA 104: 18778-18783.

Gais S, Rasch B, Wagner U, Born J (2008). Visual-procedural memory consolidation during sleep blocked by glutamatergic receptor antagonists. J Neurosci 28: 5513-5518.

Hardt O, Migues PV, Hastings M, Wong J, Nader K (2010). PKMzeta maintains 1-day- and 6-day-old long-term object location but not object identity memory in dorsal hippocampus. Hippocampus 20: 691-695.

Hardt O, Nader K, Nadel L (2013). Decay happens: the role of active forgetting in memory. Trends Cogn Sci 17: 111-120.

Hartvig P, Valtysson J, Lindner KJ, Kristensen J, Karlsten R, Gustafsson LL et al (1995). Central nervous system effects of subdissociative doses of (S)-ketamine are related to plasma and brain concentrations measured with positron emission tomography in healthy volunteers. Clin Pharmacol Ther 58: 165-173.

Herman JP, Mueller NK, Figueiredo H (2004). Role of GABA and glutamate circuitry in hypothalamo-pituitary-adrenocortical stress integration. Ann NY Acad Sci 1018: 35-45.

Hoddes E, Zarcone V, Smythe H, Phillips R, Dement WC (1973). Quantification of sleepiness: a new approach. Psychophysiology 10: 431-436.

Hrabetova S, Sacktor TC (2001). Transient translocation of conventional protein kinase $\mathrm{C}$ isoforms and persistent downregulation of atypical protein kinase Mzeta in long-term depression. Brain Res Mol Brain Res 95: 146-152.

Inostroza M, Born J (2013). Sleep for preserving and transforming episodic memory. Annu Rev Neurosci 36: 79-102. 
Janke W, Debus G (1978). Die Eigenschaftswörterliste EWL. Hogrefe: Göttingen, Germany.

Ji D, Wilson MA (2007). Coordinated memory replay in the visual cortex and hippocampus during sleep. Nat Neurosci 10: 100-107.

Kalisch R, Holt B, Petrovic P, De Martino B, Kloppel S, Buchel C et al (2009). The NMDA agonist D-cycloserine facilitates fear memory consolidation in humans. Cereb Cortex 19: 187-196.

Karni A, Meyer G, Rey-Hipolito C, Jezzard P, Adams MM, Turner R et al (1998). The acquisition of skilled motor performance: fast and slow experience-driven changes in primary motor cortex. Proc Natl Acad Sci USA 95: 861-868.

King C, Henze DA, Leinekugel X, Buzsaki G (1999). Hebbian modification of a hippocampal population pattern in the rat. J Physiol 521(Pt 1): 159-167.

Kochlamazashvili G, Bukalo O, Senkov O, Salmen B, Gerardy-Schahn R, Engel AK et al (2012). Restoration of synaptic plasticity and learning in young and aged NCAMdeficient mice by enhancing neurotransmission mediated by GluN2A-containing NMDA receptors. J Neurosci 32: 2263-2275.

Kuriyama K, Honma M, Koyama S, Kim Y (2011a). D-cycloserine facilitates procedural learning but not declarative learning in healthy humans: a randomized controlled trial of the effect of D-cycloserine and valproic acid on overnight properties in the performance of non-emotional memory tasks. Neurobiol Learn Mem 95: 505-509.

Kuriyama K, Honma M, Shimazaki M, Horie M, Yoshiike T, Koyama S et al (2011b). An N-methyl-D-aspartate receptor agonist facilitates sleep-independent synaptic plasticity associated with working memory capacity enhancement. Sci Rep 1: 127.

Kuriyama K, Honma M, Soshi T, Fujii T, Kim Y (2011c). Effect of $\mathrm{D}$-cycloserine and valproic acid on the extinction of reinstated fear-conditioned responses and habituation of fear conditioning in healthy humans: a randomized controlled trial. Psychopharmacology (Berl) 218: 589-597.

Lang PJ, Bradley MM, Cuthbert BN (2008). International affective picture system (IAPS): affective ratings of pictures and instruction manual. Technical Report A-8. University of Florida: Gainesville, FL.

Lee JL, Milton AL, Everitt BJ (2006). Reconsolidation and extinction of conditioned fear: inhibition and potentiation. J Neurosci 26: 10051-10056.

Liu L, Wong TP, Pozza MF, Lingenhoehl K, Wang Y, Sheng M et al (2004). Role of NMDA receptor subtypes in governing the direction of hippocampal synaptic plasticity. Science 304: 1021-1024.

Malenka RC, Bear MF (2004). LTP and LTD: an embarrassment of riches. Neuron 44: 5-21.

Malenka RC, Nicoll RA (1999). Long-term potentiation-a decade of progress? Science 285: 1870-1874.

Marshall L, Helgadottir H, Molle M, Born J (2006). Boosting slow oscillations during sleep potentiates memory. Nature 444: 610-613.

Marshall L, Molle M, Hallschmid M, Born J (2004). Transcranial direct current stimulation during sleep improves declarative memory. J Neurosci 24: 9985-9992.

Migues PV, Hardt O, Wu DC, Gamache K, Sacktor TC, Wang YT et al (2010). PKMzeta maintains memories by regulating GluR2-dependent AMPA receptor trafficking. Nat Neurosci 13: 630-634.

Mölle M, Born J (2011). Slow oscillations orchestrating fast oscillations and memory consolidation. Prog Brain Res 193: 93-110.
Nader K, Hardt O (2009). A single standard for memory: the case for reconsolidation. Nat Rev Neurosci 10: 224-234.

O'Neill J, Pleydell-Bouverie B, Dupret D, Csicsvari J (2010). Play it again: reactivation of waking experience and memory. Trends Neurosci 33: 220-229.

Onur OA, Schlaepfer TE, Kukolja J, Bauer A, Jeung H, Patin A et al (2010). The N-methyl-D-aspartate receptor co-agonist D-cycloserine facilitates declarative learning and hippocampal activity in humans. Biol Psychiatry 67: 1205-1211.

Oudiette D, Paller KA (2013). Upgrading the sleeping brain with targeted memory reactivation. Trends Cogn Sci 17: 142-149.

Plihal W, Born J (1997). Effects of early and late nocturnal sleep on declarative and procedural memory. J Cogn Neurosci 9: 534-547.

Rasch B, Buchel C, Gais S, Born J (2007). Odor cues during slowwave sleep prompt declarative memory consolidation. Science 315: 1426-1429.

Rechtschaffen A, Kales A (1968). A Manual of Standardized Terminology, Technique and Scoring System for Sleep Stages of Human Sleep. Brain Information Service, Brain Information Institute, UCLA: Los Angeles, CA, USA.

Ribeiro S, Shi X, Engelhard M, Zhou Y, Zhang H, Gervasoni D et al (2007). Novel experience induces persistent sleep-dependent plasticity in the cortex but not in the hippocampus. Front Neurosci 1: 43-55.

Rosenzweig ES, Barnes CA, McNaughton BL (2002). Making room for new memories. Nat Neurosci 5: 6-8.

Rudoy JD, Voss JL, Westerberg CE, Paller KA (2009). Strengthening individual memories by reactivating them during sleep. Science 326: 1079.

Sheinin A, Shavit S, Benveniste M (2001). Subunit specificity and mechanism of action of NMDA partial agonist D-cycloserine. Neuropharmacology 41: 151-158.

Siapas AG, Wilson MA (1998). Coordinated interactions between hippocampal ripples and cortical spindles during slow-wave sleep. Neuron 21: 1123-1128.

Skaggs WE, McNaughton BL (1996). Replay of neuronal firing sequences in rat hippocampus during sleep following spatial experience. Science 271: 1870-1873.

Tononi G, Cirelli C (2006). Sleep function and synaptic homeostasis. Sleep Med Rev 10: 49-62.

Van Der Werf YD, Altena E, Schoonheim MM, Sanz-Arigita EJ, Vis JC, De Rijke W et al (2009). Sleep benefits subsequent hippocampal functioning. Nat Neurosci 12: 122-123.

Villarreal DM, Do V, Haddad E, Derrick BE (2002). NMDA receptor antagonists sustain LTP and spatial memory: active processes mediate LTP decay. Nat Neurosci 5: 48-52.

Wagner U, Gais S, Born J (2001). Emotional memory formation is enhanced across sleep intervals with high amounts of rapid eye movement sleep. Learn Mem 8: 112-119.

Walker MP, Brakefield T, Seidman J, Morgan A, Hobson JA, Stickgold R (2003). Sleep and the time course of motor skill learning. Learn Mem 10: 275-284.

Watson D, Clark LA, Tellegen A (1988). Development and validation of brief measures of positive and negative affect: the PANAS scales. J Pers Soc Psychol 54: 1063-1070.

Wilson MA, McNaughton BL (1994). Reactivation of hippocampal ensemble memories during sleep. Science 265: 676-679.

Zhu M, Nix DE, Adam RD, Childs JM, Peloquin CA (2001). Pharmacokinetics of cycloserine under fasting conditions and with high-fat meal, orange juice, and antacids. Pharmacotherapy 21: 891-897.

Supplementary Information accompanies the paper on the Neuropsychopharmacology website (http://www.nature.com/npp) 